\title{
Chemometric and Microscopic Analyses for the Size Growth of Monolayer-Protected Gold Nanoparticles during their Superlattice Formation
} Hiroshi Yao, ${ }^{*}$ Keita Moriyama, and Keisaku Kimura

Graduate School of Material Science, University of Hyogo, 3-2-1 Koto, Kamigori-cho, Ako-gun, Hyogo

678-1297, Japan

\section{Abstract Spectra of Sample-L obtained by PCA}

To resolve minor spectral components that caused the change in the absorption profiles of the sample-L, PCA was performed also for this sample. The first three PCA loadings are presented as abstract spectra in Figure S1. All the abstract spectra were multiplied by -1 . The first PCA loading reasonably yielded the average spectrum, implying that the major absorption spectral change is governed by the concentration fluctuation. The second PCA loading had an almost identical profile with the first abstract spectrum in the long wavelength region, whereas that in the short wavelength side $(<\sim 250 \mathrm{~nm})$ differed. See the inset in Figure S1-b that shows the normalized spectra of the first-second PCA loadings. Since NAG exhibits electronic absorption only at $<\sim 250 \mathrm{~nm}$, the behavior would be due to the fluctuation from the NAG moiety, and complete similarity in the long wavelength region suggests the absence of size change of the gold core. The third PCA loading might imply some changes in the metal-based transition region.
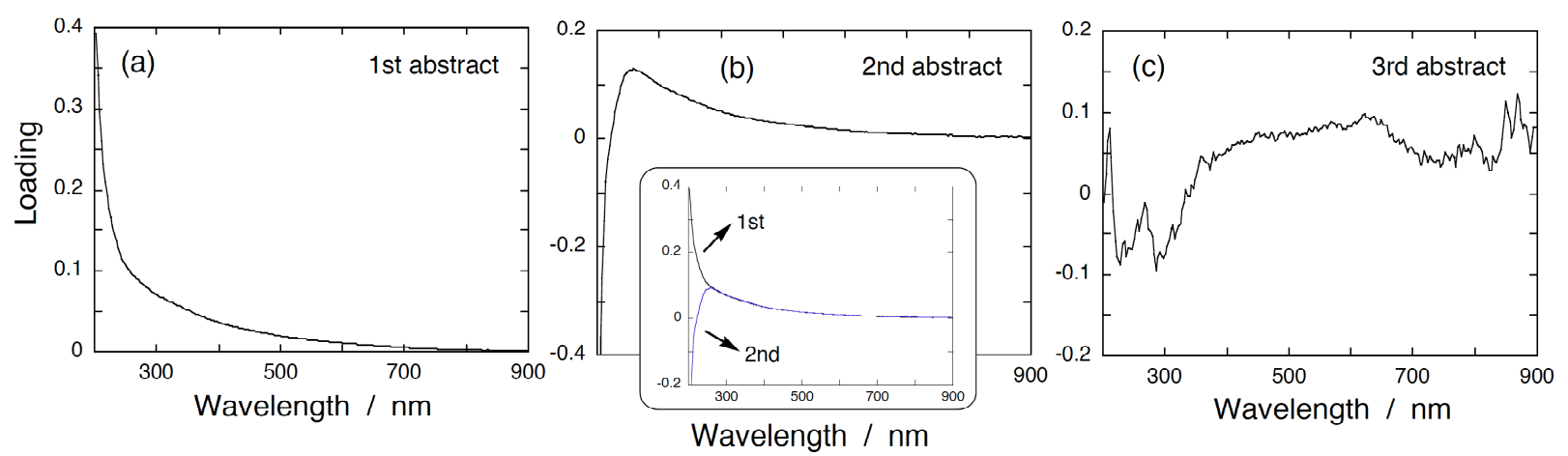

Figure S1. (a) First, (b) second, and (c) third abstract spectra yielded by PCA based on the absorption spectra shown in Figure $2 \mathrm{~b}$ in the main text. 
We calculated the eigenvalues that correspond to the first-third loadings to be $29.5,3.3 \times 10^{-1}$, and $7.5 \times 10^{-4}$, respectively. This indicates that the ratio of the intensity-change scale in the first-third loadings is approximately 198/21/1. Therefore, the core size change originated from the third abstract spectrum may have occurred in the sample-L (and this change may correlate with that observed in Figure 4c in the main text); however, comparing between the eigenvalues for the second and third PCA loadings, the latter would make very little contribution (one should bear in mind that the normalized spectral patterns were almost completely identical with each other.), and thus led to no superlattice formation of grown nanoparticles. 